\title{
Análisis del síndrome de burnout en docentes de educación infantil, primaria y secundaria para su prevención y tratamiento
}

\author{
Analysis of Burnout Syndrome in Teachers of Preschool, Middle School and High School for \\ Prevention and Treatment
}

\section{Análise da síndrome de burnout em professores do ensino pré-escolar, fundamental e médio, para prevenção e tratamento}

José Alberto Gallardo-López

Universidad Pablo de Olavide

Sevilla, España

jagallop@upo.es

http://orcid.org/0000-0003-3243-1676

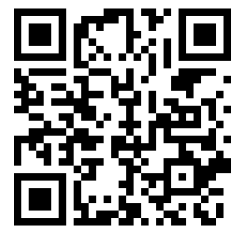

Fernando López-Noguero

Universidad Pablo de Olavide

Sevilla, España

flopnog@upo.es

http://orcid.org/0000-0002-1124-8613

Pedro Gallardo-Vázquez

Universidad de Sevilla

Sevilla, España

pgallardo@us.es

http://orcid.org/0000-0001-9703-0502

Recibido • Received • Recebido: 06 / 05 / 2018

Corregido • Revised • Revisado: 01 / 12 / 2018

Aceptado • Accepted • Aprovado: 14/ 03 / 2019

\begin{abstract}
Resumen: Este artículo presenta los resultados de una investigación realizada sobre diversos aspectos del síndrome de burnout y su incidencia en docentes de infantil, primaria y secundaria de la provincia de Sevilla (España). El objetivo principal del estudio es analizar el nivel de burnout que muestra el personal docente, tanto a nivel general como teniendo en cuenta las variables categóricas establecidas en función de la edad, los años de experiencia, el tipo de centro educativo y el nivel de enseñanza que imparten. La metodología es de carácter cuantitativo y la muestra del estudio está constituida por 1890 docentes, a quienes se aplicó la escala de burnout del cuestionario CBP-R. Para la selección de la muestra se siguieron los criterios establecidos por Sierra (1987), obteniendo un alto índice de fiabilidad cercano al +/- 2\% de margen de error. El análisis de los datos y el tratamiento de los resultados obtenidos se llevó a cabo teniendo en cuenta variables como el agotamiento emocional, la despersonalización y la falta de realización, y en función de la edad, los años de experiencia docente, el tipo de centro donde trabajan y el nivel de enseñanza que imparten.
\end{abstract}


doi: http://dx.doi.org/10.15359/ree.23-2.17

URL: http://www.una.ac.cr/educare

CORREO: educare@una.cr

Entre los principales hallazgos destacamos que un 11,6\% de docentes que se encuestaron presenta un nivel alto de burnout general. De manera específica, sobre el agotamiento emocional, el $74 \%$ obtuvo un puntaje dentro del nivel medio de burnout y destaca especialmente el gasto emocional de la profesión docente y lo duro del síndrome postvacacional. Sobre la despersonalización, el $83 \%$ también obtuvo una puntuación dentro del nivel medio, y todo parece indicar que el grupo docente encuestado acus $\mathrm{n}$ de forma extraordinaria la presión en su trabajo. Por último, atendiendo a la falta de realización, en la que el 86,2 \% obtuvo una puntuación de nivel medio de burnout, podemos decir que se aprecia mucha satisfacción acerca del trabajo que desempeñan y su identificación vocacional.

Palabras claves: Docencia; burnout; condiciones de empleo del docente; escala de burnout del cuestionario CBP-R; situación docente.

Abstract: This article presents the results of a research project based on various aspects of the burnout syndrome and its incidence in teachers of preschool, middle school, and high school from Seville province (Spain). The main goal of the study is to analyze the level of burnout that teachers experience both at a general level and when considering different variables according to age, experience, type of educational center and educational stage. A quantitative methodology was implemented, and the study sample was composed of 1890 teachers, to whom the CBP-R Burnout scale questionnaire was applied. Sierra's (1987) criteria were used to select the sample; a high-reliability index close to $+/-2 \%$ margin of error was obtained. For the data analysis and the treatment of the obtained results, variables such as emotional exhaustion, depersonalization and lack of realization were taken into consideration, as well as variables according to age, experience, type of educational center and educational level. Among the main findings, we highlight that $11.6 \%$ of teachers surveyed have a high level of general burnout. In terms of emotional exhaustion, $74 \%$ of them obtained a score within the burnout average level, and especially highlighted the emotional cost of the education profession and the harshness of the post-holiday syndrome. About the depersonalization, $83 \%$ also obtained a score within the average level; everything seems to indicate that the teachers surveyed suffer from an extraordinary form of stress at their jobs. At last, in terms of the lack of realization in their profession, $86.2 \%$ of the teachers surveyed obtained an average burnout level; we can appreciate that there is a high satisfaction doing their jobs and with their professional vocation too.

Keywords: teaching; burnout; conditions of teacher employment; burnout scale of the CBP-R questionnaire; teacher situation.

Resumo: Este artigo apresenta os resultados de uma pesquisa realizada sobre diversos aspectos da síndrome de burnout e sua incidência no corpo docente de crianças eestudantes do ensinofundamental e médio na província de Sevilha (Espanha). O principal objetivo do estudo é analisar o nível de burnout que mostra o pessoal docente, tanto a nível geral considerando as variáveis categóricas estabelecidas em função da idade, anos de experiência, tipo de centro educativo e o nível de ensino que lecionam. A metodologia é de caráter quantitativo e a mostra do estudo está constituída por 1890 docentes, a quem aplicou-se a escala de burnout do questionário CBP-R. Para a seleção da mostra seguiram-se os critérios estabelecidos por Serra (1987), obtendo um alto índice de confiabilidade próximo ao +/- $2 \%$ de margem de erro. A análise dos dados e o tratamento dos resultados obtidos levou-se a cabo tendo em conta variáveis como o desgaste emocional, a despersonalização e a falta de realização, e em 
Revista Electrónica Educare (Educare Electronic Journal) EISSN: 1409-4258 Vol. 23(2) MAYO-AGOSTO, 2019:1-20

doi: http://dx.doi.org/10.15359/ree.23-2.17

URL: http://www.una.ac.cr/educare

CORREO: educare@una.cr

\begin{abstract}
função da idade, dos anos de experiência docente, o tipo de centro onde trabalham e o nível de ensino que oferecem. Entre os principais resultados, destacamos que $11,6 \%$ dos professores pesquisados tiveram um alto nível de burnout geral. Especificamente, sobre o desgaste emocional, $74 \%$ obtiveram uma pontuação dentro do nível médio de burnout e destaca especialmente o consumo emocional da profissão docente e a aspereza da síndrome pós-férias. Na despersonalização, 83 \% também obtiveram pontuação dentro do nível médio, e tudo parece indicar que o grupo de professores participantes foi extraordinariamente pressionado no seu trabalho. Finalmente, considerando a falta de desempenho, em que $86,2 \%$ obteve uma pontuação média de burnout, podemos dizer que há muita satisfação sobre o trabalho que realizam e sua identificação profissional.
\end{abstract}

Palavras-chave: Ensino; burnout; condições de trabalho do corpo docente; escala de burnout do questionário CBP-R; situação de ensino.

\title{
Introducción
}

Vivimos una época de cambios acelerados y fenómenos de nuevo cuño tales como la globalización, la transición demográfica, el impacto de las nuevas tecnologías y las cambiantes relaciones de trabajo en los mercados laborales que están influyendo psicológicamente en la vida profesional de las personas. En efecto, los puestos de trabajo cambian, y con ellos cambian también las exigencias y presiones que tienen que afrontar diariamente. El hecho de que muchas personas desarrollen síntomas del denominado "síndrome del quemado" o de "desgaste profesional" (burn-out, en inglés), subraya la necesidad de tomar en serio los factores psicosociales que actúan en el lugar de trabajo.

Burnout significa literalmente "quemado" y se utiliza, cuando se habla de estrés laboral, para describir una situación de total agotamiento o fatiga. Es un estado de desgaste físico, mental y emocional que puede traer como consecuencia el absentismo laboral, el abandono del puesto de trabajo o la incapacidad total para volver a trabajar.

Herbert J. Freudenberger, psicólogo estadounidense, fue el primero que acuñó el término de "burnout" en el sentido que nos ocupa de "agotamiento profesional". Este autor define dicho término como la extinción de la motivación o el incentivo, especialmente allí donde la dedicación a un objetivo o a una relación fracasa en conseguir los resultados deseados (Freudenberger, 1974).

Este síndrome se caracteriza por una pérdida de ilusión por el trabajo, desgaste psíquico e indolencia y se produce cuando las exigencias del entorno laboral sobrepasan la capacidad de la persona para afrontarlas. Esta situación se da cuando la persona presenta episodios frecuentes de cansancio emocional intenso en relación con su trabajo, actitudes y sentimientos negativos hacia los demás individuos y despersonalización. 
doi: http://dx.doi.org/10.15359/ree.23-2.17

URL: http://www.una.ac.cr/educare

CORREO: educare@una.cr

Las situaciones que provocan la aparición del síndrome de burnout son de gran magnitud y persistentes hasta provocar síntomas tales como cambios de estado de ánimo, desmotivación, agotamiento mental, falta de energía y menor rendimiento, así como afectaciones emocionales, despersonalización o falta de realización personal, entre otras (Góngora, Pérez y Torres, 2016).

Este síndrome no solo afecta a nivel psicológico, sino que también afecta a nivel físico al provocar alteraciones del sistema locomotor y otras alteraciones psicosomáticas tales como problemas gástricos y cardiovasculares, mareos, cefaleas, etc.

De igual forma, debemos señalar que el síndrome de burnout no afecta solo al individuo, sino que es un proceso multidimensional influenciado por factores de índole interpersonal, organizacional, etc.

Sobre el burnout en ámbitos educativos, podemos destacar los estudios de Moreno, Garrosa y González (2000) sobre la evaluación del estrés y el burnout del profesorado, los de Kroupis et al. (2017), Manassero, Vázquez, Ferrer, Fornés y Fernández (2003), sobre estrés y burnout en la enseñanza, así como los de Boyd y Schneider (2014), sobre el burnout de personas cuidadoras infantiles o el de Solera, Gutierrez y Palacios-Ceña (2017) sobre implicaciones psicológicas del burnout.

Bilge (2006), por su parte, analiza el burnout del personal académico en relación con la satisfacción laboral y otros factores, mientras Rodríguez y Fernández $(2012,2017)$ estudian en sus trabajos el burnout en el profesorado de secundaria y su relación con variables personales y profesionales, junto a los de Zamora-Díaz, Cobos-Sanchiz y López-Noguero (2017) y ZamoraDíaz, López-Noguero y Cobos-Sanchiz (2016), sobre las condiciones sociales y salud laboral del personal docente, con gran énfasis en la cuestión del burnout.

De igual forma, destacamos los trabajos de Extremera, Fernández-Berrocal y Durán (2003) así como los de Pena y Extremera (2012), Shami, Tareh y Taran (2017), centrados en la inteligencia emocional y el burnout en docentes, así como el de Botella, Longás y Gómez (2007) que aborda, con acierto, la construcción social del burnout en la profesión docente.

Finalmente, debemos señalar en este punto, que en la cuestión del burnout docente es fundamental tanto la cuestión de la prevención como la del tratamiento, situaciones donde finalmente destacamos las aportaciones de Fuertes, Aranda y Arroyo (2016), Fidalgo (2005), Gil (2003), Mameli y Molinari (2017), o Langher, Caputo y Ricci (2017).

\section{Marco teórico}

\section{Definición de burnout}

El síndrome del profesional quemado, también conocido como desgaste profesional o burnout, se refiere "a un conjunto de síntomas que persisten y/o aumentan a lo largo del tiempo 
cuya presencia está directamente relacionada con la actividad laboral de la persona" (Gutiérrez, Atienza y García-Moya, 2015, p. 119).

En este sentido, algunas de las definiciones más aceptadas del síndrome del burnout pasan por señalar que se trata de una manifestación caracterizada por agotamiento emocional, despersonalización y reducida realización personal, así como destacar que hablamos de "un estado de agotamiento físico, emocional y mental causado por estar implicada la persona durante largos periodos de tiempo en situaciones que le afectan emocionalmente" (Pines y Aronson, 1998, p. 9).

Por su parte, Gil (2001, p. 2) lo define en los siguientes términos:

Una respuesta al estrés laboral crónico integrado por actitudes y sentimientos negativos hacia las personas con las que se trabaja [despersonalización] y hacia el propio rol profesional [falta de realización personal en el trabajo], así como por la vivencia de encontrarse emocionalmente agotado [agotamiento emocional].

En opinión de este autor, el síndrome de burnout no debe identificarse únicamente con el estrés psicológico, sino que debe ser entendido como una respuesta a las fuentes de estrés crónico (Gil, 2001).

Las características esenciales del síndrome de burnout son las siguientes: 1) agotamiento físico, mental y emocional; 2) sentimiento de inadecuación, que surge como consecuencia de la evaluación negativa que hace la persona de sí misma, que siente que no dispone de la capacidad necesaria para afrontar y superar los requerimientos de su actividad laboral; 3 ) despersonalización, que se manifiesta en actitudes y respuestas negativas hacia las personas con las que trabaja; y 4) falta de implicación en los problemas y pérdida de motivación laboral.

En cualquier caso, definimos el síndrome de burnout como el desgaste profesional que sufren las personas que trabajan en los servicios sanitarios, educativos, sociales, etc., al estar expuestas a demandas emocionales elevadas. Obviamente, hacemos referencia a un problema humano y social de primera línea en la sociedad actual que necesita, como tantos otros, de análisis y estrategias de intervención (López-Noguero, 2015).

\section{Etiología del burnout}

El síndrome de burnout es un proceso multicausal y altamente complejo donde, entre sus causas destacan: aburrimiento y estrés, exceso de trabajo, pobres condiciones económicas, falta de estimulación, escasa orientación profesional, aislamiento y crisis en el desarrollo de la carrera profesional (Ledesma, 2015). 
doi: http://dx.doi.org/10.15359/ree.23-2.17

URL: http://www.una.ac.cr/educare

CORREO: educare@una.cr

Las causas del síndrome de burnout entre el profesorado son muy diversas, "pero existe consenso al considerarlo como una consecuencia de la exposición a factores laborales estresantes que exceden los recursos" (Arón y Milicic, 2000; Durán, Extemera y Rey, 2001; Gil-Monte y Peiró 1999; Maslach y Leiter, 2005, citados por Pena y Extremera, 2012, p. 607) y capacidades de las personas. Al respecto, en la bibliografía científica se han establecido diferentes categorías causales que influyen en la aparición y mantenimiento del burnout en el colectivo docente, [a saber:] 1) la relación directa conel alumnado; ... 2) los factores organizacionales; ... y 3 ) los factores personales (Manassero, Fornés, Fernández, Vázquez y Ferrer, 2003; Moriana y Herruzo, 2004, citados por Pena y Extremera, 2012, p. 607). En cualquier caso, a lo largo de "los últimos años, se está prestando más atención a las diferencias individuales y a los recursos personales como factores que previenen el [síndrome de] burnouty sus consecuencias negativas" (Extremera, Durán y Rey, 2007; Palomera, Gil-Olarte y Brackett, 2006; Schauffeli y Bakker, 2004, citados por Pena y Extremera, 2012, pp. 607-608). A estas causas, podríamos añadirle otras tales como: "problemas de disciplina, ... falta de apoyo de pares y personal directivo, ... introversión, ... falta de autoridad, sobrecarga de trabajo, ... tendencia a la neurosis, escasa motivación por el aprendizaje, presiones de tiempo, falta de autoestima, escasez de recursos, sueldo insuficiente, falta de reconocimiento profesional, etc. (Manassero, Fornés, Fernández, Vàzquez y Ferrer, 1995; Moriana y Herruzo, 2004, citados por Pena y Extremera, 2012).

\section{Metodología}

El objetivo fundamental de esta investigación es medir el nivel de burnout en los docentes de infantil, primaria y secundaria de los centros públicos y privados de la provincia de Sevilla y, para ello, la metodología de investigación utilizada en este trabajo ha sido de carácter cuantitativo, con un diseño no experimental, de tipo exploratorio, transversal y correlacional, enmarcado dentro de los estudios ex-post-facto.

\section{Participantes}

La población perteneciente a docentes que imparten clases en centros públicos y privados de educación infantil, primaria y secundaria de la provincia de Sevilla (España) es de un total de 27534 (Instituto de Estadística y Cartografía de Andalucía, 2016).

Para la selección de la muestra hemos seguido los criterios establecidos por Sierra (1987), en los que se indica que, para una población de 20000 personas se establece un margen de error $+/-3 \%$ (1053) y $+/-2 \%$ (2222). Teniendo en cuenta que la muestra seleccionada es de 1890 docentes, que imparten clases en centros educativos públicos y privados, estaríamos en un alto índice de fiabilidad, acercándonos más al +/- $2 \%$ de margen de error. De la muestra seleccionada, 270 ejercen la docencia en educación infantil (alumnado de 0 a 6 años), 400 en educación primaria (alumnado de 6 a 12 años) y 1220 en institutos de educación secundaria (alumnado de 12 a 18 años). 


\section{Instrumento}

Para medir el nivel de burnout en docentes de los centros educativos públicos y privados de la provincia de Sevilla se utilizó el instrumento diseñado por Moreno et al. (2000), denominado Cuestionario de burnout del profesorado (CBP-R), versión revisada a partir de versiones previas, de acuerdo con Solera et al. (2017). Este cuestionario se compone de tres factores: Factor I (estrés y burnout), que se refiere, por un lado, al estrés de rol laboral, y por otra parte, a cuestiones relativas al proceso de burnout y de cada una de sus dimensiones (agotamiento emocional, despersonalización, y falta de realización); factor II (desorganización), que hace referencia a las condiciones en las que se realiza el trabajo, al estilo de dirección, y al apoyo recibido por parte de quien supervisa; y factor III (problemática administrativa), que se refiere a las preocupaciones profesionales y al reconocimiento profesional.

El CBP-R está compuesto por 66 ítems que se responden mediante una escala de tipo Likert formada por 5 puntos (desde $1=$ no me afecta, hasta $5=$ me afecta muchísimo, para los 11 primeros ítems; desde 1 = totalmente en desacuerdo, hasta 5 = totalmente de acuerdo, para los 55 ítems restantes).

Las valoraciones más altas que se han obtenido en la escala de burnout y en cada una de las subescalas que la componen se relacionan, de manera teórica, con una mayor problemática para el personal docente en relación con las variables analizadas.

En el estudio solo se utilizaron los ítems de la escala de burnout del cuestionario CBP-R. Por tanto, en el análisis estadístico y tratamiento de los datos se incluirá una tabla por cada subescala o variable estudiada perteneciente a la escala burnout.

\section{Procedimiento}

Previo permiso de los autores del cuestionario CBP-R para su utilización en el presente estudio, se aplicó la escala de burnout del cuestionario CBP-R de forma autoadministrada a 1890 docentes en el tercer trimestre del curso 2016-2017; este trimestre se seleccionó de manera intencionada por ser el período en el que los cuerpos docentes pueden presentar mayores niveles de burnout (Botella et al., 2007; Rodríguez y Fernández, 2012); en quinto lugar, se realizó el análisis de los datos y los resultados obtenidos mediante el uso del paquete informático SPSS versión 24. Tras esta reducción de datos, finalmente, se obtuvieron las conclusiones.

\section{Resultados, análisis y discusión}

Como hemos señalado, el tratamiento estadístico de los datos se hizo con el paquete estadístico SPSS 24, realizando, en primer lugar, un análisis de la consistencia interna de las 
doi: http://dx.doi.org/10.15359/ree.23-2.17

URL: http://www.una.ac.cr/educare

CORREO: educare@una.cr

subescalas de la escala de burnout del cuestionario CBP-R; en segundo lugar, un análisis estadístico de sus ítems y, en tercer lugar, un estudio diferencial del síndrome de burnout en función de las variables categóricas edad, años de experiencia docente, tipo de centro y nivel de enseñanza.

\section{Consistencia interna de la escala de burnout y de sus subescalas (agotamiento emocional, despersonalización y falta de realización)}

La consistencia interna obtenida en la escala de burnout y en cada una de las subescalas que la componen, medida mediante el estadístico alfa de Cronbach, fue la siguiente: burnout: $a$ de Cronbach $=0.771$; agotamiento: $\alpha$ de Cronbach $=0.949$; despersonalización: $a=0.823$; falta de realización: $\alpha=0.770$. Todos estos valores indican que la consistencia interna para la escala de burnout y para la subescala falta de realización es aceptable $(a=0.7-0.8)$, para la subescala despersonalización es buena $(\alpha=0.8-0.9)$ y para la subescala agotamiento es excelente ( $a$ $=>0.9$ ), según las categorías propuestas por George y Mallery (2003) por lo que la fiabilidad, estimada con el alfa de Cronbach, podemos considerarla en este caso como apta y suficiente. A partir de los datos obtenidos, se tomó la decisión de no modificar la estructura del instrumento.

\section{Análisis estadístico}

A continuación, se presentan los estadísticos descriptivos (media, mediana moda, desviación estándar, varianza y rango) por ítems de cada una de las subescalas o variables que conforman la escala de burnout.

En la Tabla 1 se observan los estadísticos descriptivos por ítems de la subescala "agotamiento emocional".

Las mayores puntuaciones medias obtenidas en la subescala "agotamiento emocional", corresponden a los ítems 1 y 5 ; el primero guarda relación con la fatiga y el cansancio laboral; el segundo, con el síndrome de vuelta al trabajo o estrés post-vacacional.

En este sentido, apreciamos cómo el profesorado consultado destaca especialmente el gasto emocional de la profesión docente y lo duro del síndrome postvacacional del profesorado (Extremera et al., 2003; Pena y Extremera, 2012).

Ítem 1: Enseñar me agota emocionalmente.

Ítem 5: Se me hace muy difícil volver al trabajo después de las vacaciones.

Por su parte, el ítem con menor puntuación en la media es el 2, que guarda relación con el estrés y la tensión laboral.

Ítem 2: Siento que cualquier día podría tener un ataque de nervios, si no dejo de enseñar. 
Tabla 1: Estadísticos descriptivos por ítems de la subescala agotamiento emocional

\begin{tabular}{|c|c|c|c|c|c|}
\hline & & $\begin{array}{l}\text { Enseñar me agota } \\
\text { emocionalmente }\end{array}$ & $\begin{array}{l}\text { Siento que cualquier } \\
\text { día podría tener un } \\
\text { ataque de nervios, si } \\
\text { no dejo de enseñar }\end{array}$ & $\begin{array}{l}\text { Me siento ansioso/a } \\
\text { y tenso/a al ir a } \\
\text { trabajar cada día }\end{array}$ & $\begin{array}{l}\text { Mi profesión está afectando } \\
\text { negativamente mis } \\
\text { relaciones fuera del trabajo }\end{array}$ \\
\hline \multirow{2}{*}{$\mathrm{N}$} & Válido & 1890 & 1890 & 1890 & 1890 \\
\hline & Perdidos & 0 & 0 & 0 & 0 \\
\hline \multicolumn{2}{|c|}{ Media } & 2,53 & 1,97 & 2,05 & 2,14 \\
\hline \multicolumn{2}{|c|}{ Mediana } & 2,00 & 1,00 & 1,00 & 1,00 \\
\hline \multicolumn{2}{|c|}{ Moda } & 1 & 1 & 1 & 1 \\
\hline \multicolumn{2}{|c|}{$\begin{array}{l}\text { Desviación } \\
\text { estándar }\end{array}$} & 1,390 & 1,345 & 1,328 & 1,425 \\
\hline \multicolumn{2}{|c|}{ Varianza } & 1,932 & 1,808 & 1,764 & 2,031 \\
\hline \multicolumn{2}{|c|}{ Rango } & 4 & 4 & 4 & 4 \\
\hline \multirow{3}{*}{$\mathrm{N}$} & & $\begin{array}{l}\text { Se me hace muy } \\
\text { difícil volver al } \\
\text { trabajo después de } \\
\text { las vacaciones }\end{array}$ & $\begin{array}{l}\text { Siento que mi trabajo } \\
\text { está afectando } \\
\text { negativamente mi } \\
\text { salud }\end{array}$ & $\begin{array}{l}\text { Con frecuencia me } \\
\text { siento deprimido/a } \\
\text { respecto a mi } \\
\text { profesión }\end{array}$ & $\begin{array}{l}\text { Si un buen amigo/a } \\
\text { me dijera que estaba } \\
\text { interesado/a en tener un } \\
\text { trabajo aquí tendría serias } \\
\text { reservas en recomendárselo }\end{array}$ \\
\hline & Válido & 1890 & 1890 & 1890 & 1890 \\
\hline & Perdidos & 0 & 0 & 0 & 0 \\
\hline \multicolumn{2}{|c|}{ Media } & 2,67 & 2,43 & 2,31 & 2,29 \\
\hline \multicolumn{2}{|c|}{ Mediana } & 2,00 & 2,00 & 2,00 & 2,00 \\
\hline \multicolumn{2}{|c|}{ Moda } & 1 & 1 & 1 & 1 \\
\hline \multicolumn{2}{|c|}{$\begin{array}{l}\text { Desviación } \\
\text { estándar }\end{array}$} & 1,486 & 1,503 & 1,451 & 1,405 \\
\hline \multicolumn{2}{|c|}{ Varianza } & 2,208 & 2,259 & 2,105 & 1,974 \\
\hline \multicolumn{2}{|c|}{ Rango } & 4 & 4 & 4 & 4 \\
\hline
\end{tabular}

Nota: Elaboración propia a partir de Moreno et al. (2000). 
doi: http://dx.doi.org/10.15359/ree.23-2.17

URL: http://www.una.ac.cr/educare

CORREO: educare@una.cr

En la Tabla 2 se muestran los estadísticos descriptivos por ítems de la subescala "despersonalización".

Tabla 2: Estadísticos descriptivos por ítems de la subescala despersonalización

\begin{tabular}{|c|c|c|c|c|c|}
\hline & & $\begin{array}{l}\text { A veces tiendo a } \\
\text { tratar al estudiantado } \\
\text { como objetos } \\
\text { impersonales }\end{array}$ & $\begin{array}{l}\text { Siento que mis } \\
\text { alumnos/as son el/la } \\
\text { enemigo/a }\end{array}$ & $\begin{array}{l}\text { Siento una presión } \\
\text { constante por parte de } \\
\text { los/as otros/as para que } \\
\text { mejore mi trabajo }\end{array}$ & $\begin{array}{l}\text { Siento que realmente } \\
\text { no les gusto a mis } \\
\text { alumnos/as }\end{array}$ \\
\hline $\mathrm{N}$ & Válido & 1890 & 1890 & 1890 & 1890 \\
\hline & Perdidos & 0 & 0 & 0 & 0 \\
\hline & dia & 1,47 & 1,55 & 2,37 & 1,68 \\
\hline & diana & 1,00 & 1,00 & 2,00 & 1,00 \\
\hline & da & 1 & 1 & 1 & 1 \\
\hline & $\begin{array}{l}\text { sviación } \\
\text { ándar }\end{array}$ & ,900 & ,976 & 1,365 & 1,027 \\
\hline & rianza & 810 & 953 & 1,862 & 1,055 \\
\hline & ngo & 4 & 4 & 4 & 4 \\
\hline
\end{tabular}

Nota: Elaboración propia a partir de Moreno et al. (2000).

Lasmenores puntuacionesmedias obtenidasenla subescala despersonalización, corresponden a los ítems 1 y 2; el primero guarda relación con las actitudes negativas del profesorado hacia el alumnado; el segundo, con los sentimientos negativos de docentes hacia el alumnado, que en el caso de los grupos docentes encuestados no son los aspectos más significativos.

Ítem 1: A veces tiendo a tratar a los/as estudiantes como objetos impersonales.

Ítem 2: Siento que mis alumnos/as son "el/la enemigo/a".

Sin embargo, y desde otro punto de vista, el ítem con mayor puntuación en la media es el 3, que guarda relación con los sentimientos de presión en el trabajo, donde los sujetos docentes que cumplimentaron el cuestionario acusan de forma extraordinaria la presión en su trabajo (Zamora-Díaz et al., 2016).

Ítem 3: Siento una presión constante por parte de los/as otros/as para que mejore mi trabajo.

En la Tabla 3 se observan los estadísticos descriptivos por ítems de la subescala falta de realización. 
Tabla 3: Estadísticos descriptivos por ítems de la subescala falta de realización

\begin{tabular}{|c|c|c|c|c|c|}
\hline & & $\begin{array}{l}\text { En lo fundamental, yo } \\
\text { diría que estoy muy } \\
\text { contento/a con mi } \\
\text { trabajo }\end{array}$ & $\begin{array}{l}\text { Actualmente } \\
\text { encuentro que } \\
\text { mi vida es muy } \\
\text { provechosa }\end{array}$ & $\begin{array}{l}\text { Sabiendo lo que se } \\
\text { ahora, si tuviera que } \\
\text { decidir de nuevo si } \\
\text { elijo este trabajo, } \\
\text { definitivamente lo haría }\end{array}$ & $\begin{array}{l}\text { En general, mi } \\
\text { trabajo se adapta } \\
\text { muy bien a la clase } \\
\text { de trabajo que yo } \\
\text { deseaba }\end{array}$ \\
\hline & Válido & 1890 & 1890 & 1890 & 1890 \\
\hline 18 & Perdidos & 0 & 0 & 0 & 0 \\
\hline & dia & 3,95 & 3,76 & 3,79 & 3,64 \\
\hline & diana & 4,00 & 4,00 & 4,00 & 4,00 \\
\hline Mc & & 5 & 4 & 5 & 5 \\
\hline & $\begin{array}{l}\text { sviación } \\
\text { ándar }\end{array}$ & 1,215 & 1,138 & 1,376 & 1,348 \\
\hline & ianza & 1,476 & 1,294 & 1,894 & 1,817 \\
\hline & ngo & 4 & 4 & 4 & 4 \\
\hline & & $\begin{array}{l}\text { Siento que me es } \\
\text { imposible producir } \\
\text { algún cambio positivo } \\
\text { en la vida de mis } \\
\text { alumnos/as }\end{array}$ & $\begin{array}{l}\text { Actualmente } \\
\text { encuentro que } \\
\text { disfruto bastante } \\
\text { de mi vida }\end{array}$ & $\begin{array}{l}\text { Actualmente encuentro } \\
\text { aburrida }\end{array}$ & ni vida bastante \\
\hline & Válido & 1890 & 1890 & 18 & \\
\hline & Perdidos & 0 & 0 & & 0 \\
\hline & & 2,09 & 3,73 & 1, & \\
\hline & diana & 2,00 & 4,00 & 2, & \\
\hline & & 1 & 4 & & 1 \\
\hline & sviación & 1,143 & 1,128 & 1,1 & \\
\hline & ianza & 1,306 & 1,272 & 1,3 & \\
\hline & go & 4 & 4 & & 4 \\
\hline
\end{tabular}

Nota: Elaboración propia a partir de Moreno et al. (2000).

Esta subescala tiene puntuaciones bastante significativas. En este sentido, las mayores puntuaciones medias obtenidas en la subescala "falta de realización", corresponden a los ítems 1 y 3; el primero guarda relación con la satisfacción en el trabajo; el segundo, con la elección y desempeño de la profesión docente, donde se aprecia mucha satisfacción docente acerca de sus respectivos trabajos y vocación, muy en la línea de trabajos y estudios similares. 
doi: http://dx.doi.org/10.15359/ree.23-2.17

URL: http://www.una.ac.cr/educare

CORREO: educare@una.cr

Ítem 1: En lo fundamental, yo diría que estoy muy contento/a con mi trabajo.

Ítem 3: Sabiendo lo que sé ahora, si tuviera que decidir de nuevo si elijo este trabajo, definitivamente lo haría.

Por el contrario, el ítem con menor puntuación en la media es el 2, que guarda relación con el agrado y utilidad de la profesión de docente, que es considerada como mejorable, a pesar de la satisfacción y vocación que destaca el propio profesorado.

Ítem 2: Actualmente encuentro que mi vida es muy provechosa.

A continuación, se muestran los estadísticos (mediana, media, desviación estándar y rango posible) de la escala de burnout y de las subescalas que la componen (agotamiento emocional, despersonalización y falta de realización).

La puntuación media de la escala de burnout fue de 2,54 (desviación estándar = 1,26; rango 19-95) y la mediana de 2,21. En la subescala agotamiento emocional la media fue de 2,29 (desviación estándar $=1,41$; rango 8-40) y la mediana, de 1,62; en la subescala despersonalización la media fue de 1,76 (desviación estándar = 1,06; rango 4-20) y la mediana de 1,25; en la subescala falta de realización la media fue de 3,27 (desviación estándar = 1,21; rango 7-35) y la mediana de 3,42.

En estos datos, se aprecia que la subescala "Falta de realización" es la dimensión que más preocupa a los grupos docentes, donde la que tiene puntuaciones más elevadas resalta la complejidad de la labor docente, constantemente expuesta a situaciones estresantes y que deriva en una sensación de frustración y falta de realización personal muy grande.

En la Tabla 4 se presentan los rangos establecidos para la interpretación de las puntuaciones de la escala de burnout y de las subescalas que la conforman (agotamiento emocional, despersonalización y falta de realización). Los rangos se calcularon sumando y restando una desviación estándar a la media.

Tabla 4: Rangos establecidos para la interpretación de las puntuaciones de la escala de burnout y de sus subescalas (agotamiento emocional, despersonalización y falta de realización)

\begin{tabular}{lcccc}
\hline Escala & -1 Desviación estándar & Media & +1 Desviación estándar & Desviación estándar \\
\hline Burnout & 1,28 & 2,54 & 3,8 & 1,26 \\
\hline Subescala & -1 Desviación estándar & Media & +1 Desviación estándar & Desviación estándar \\
\hline Agotamiento emocional & 0,88 & 2,29 & 3,7 & 1,41 \\
\hline Despersonalización & 0,7 & 1,76 & 2,82 & 1,06 \\
\hline Falta de realización & 2,06 & 3,27 & 4,48 & 1,21 \\
\hline
\end{tabular}

Nota: Elaboración propia a partir de Cárdenas, Méndez y González (2014). 
El rango medio de las puntuaciones de la escala de burnout es $1,28-3,8$, de las subescalas que la forman es: agotamiento emocional $(0,88-3,7)$, despersonalización $(0,7-2,82)$ y falta de realización $(2,06-4,48)$.

En la Tabla 5 se muestran los rangos, frecuencias y porcentajes de la variable burnout (agotamiento emocional, despersonalización y falta de realización).

Tabla 5: Rangos, frecuencias y porcentajes de la variable burnout

\begin{tabular}{|c|c|c|c|}
\hline Escala & Rangos & Frecuencia & $\%$ \\
\hline & Puntaje menor al esperado ( 1,28 o menos) & 0 & 0 \\
\hline \multirow[t]{2}{*}{ Burnout } & Puntaje en el rango esperado $(1,29$ a 3,8$)$ & 1670 & 88,3 \\
\hline & Puntaje mayor al esperado (3,81 o más) & 220 & 11,6 \\
\hline \multirow[t]{2}{*}{ Subescala } & Rangos & Frecuencia & $\%$ \\
\hline & Puntaje menor al esperado $(0,88$ o menos) & 0 & 0 \\
\hline \multirow[t]{3}{*}{ Agotamiento emocional } & Puntaje en el rango esperado $(0,89$ a 3,7$)$ & 1400 & 74 \\
\hline & Puntaje mayor al esperado (3,71 o más) & 490 & 25,9 \\
\hline & Puntaje menor al esperado ( 0,7 o menos) & 0 & 0 \\
\hline \multirow[t]{3}{*}{ Despersonalización } & Puntaje en el rango esperado $(0,71$ a 2,82$)$ & 1570 & 83 \\
\hline & Puntaje mayor al esperado (2,83 o más) & 320 & 16,9 \\
\hline & Puntaje menor al esperado ( 2,06 o menos) & 260 & 13,7 \\
\hline \multirow[t]{2}{*}{ Falta de realización } & Puntaje en el rango esperado $(2,07$ a 4,48$)$ & 1630 & 86,2 \\
\hline & Puntaje mayor al esperado (4,49 o más) & 0 & 0 \\
\hline
\end{tabular}

Nota: Elaboración propia a partir de Cárdenas et al. (2014).

En la escala de burnout, el $88,3 \%$ de docentes obtuvo un puntaje dentro del rango esperado (nivel medio de burnout), el 11,6\% un puntaje mayor al rango esperado (nivel alto) y ningún docente obtuvo un puntaje menor al rango esperado (nivel bajo). En la subescala agotamiento emocional, el $74 \%$ de docentes obtuvo un puntaje dentro del rango esperado, el $25,9 \%$ un puntaje mayor al rango esperado (nivel alto) y nadie obtuvo un puntaje menor al rango esperado (nivel bajo). En la subescala despersonalización, el $83 \%$ de docentes obtuvo una puntuación dentro del rango esperado (nivel medio), el 16,9 \% una puntuación mayor al rango esperado (nivel alto) y nadie obtuvo una puntuación menor al rango esperado (nivel bajo). En la subescala falta de realización, el $86,2 \%$ de docentes obtuvo una puntuación dentro del rango esperado (nivel medio), el 13,79 \% una puntuación menor al rango esperado (nivel bajo) y nadie obtuvo una puntuación mayor al rango esperado (nivel alto). 
doi: http://dx.doi.org/10.15359/ree.23-2.17

URL: http://www.una.ac.cr/educare

CORREO: educare@una.cr

En la escala de burnout y en las subescalas agotamiento emocional, despersonalización y falta de realización, la mayor parte de docentes obtuvo un puntaje dentro del rango esperado (nivel medio de burnout); nadie obtuvo un puntaje menor al rango esperado en la escala de burnout y en todas sus subescalas, excepto en la subescala falta de realización, en la que el $13,7 \%$ de docentes obtuvo un puntaje menor a dicho rango (nivel bajo de burnout).

El agotamiento emocional presenta más puntajes en el rango mayor al esperado que la escala de burnout y las subescalas despersonalización y falta de realización.

\section{Análisis diferenciales del burnout}

A continuación, se analiza la existencia o no de diferencia en el síndrome (tomando la escala de burnout y cada una de sus subescalas) en función de las variables categóricas edad, años de experiencia docente, tipo de centro y nivel de enseñanza. Para el estudio diferencial de variables se llevó a cabo el análisis de varianza (ANOVA de un factor) y, en el supuesto de encontrar diferencias significativas, se aplicó la prueba de Scheffé para realizar comparaciones múltiples de las medias.

Tras el análisis de las desigualdades que existen en el nivel de burnout, teniendo en cuenta la edad de los grupos de docentes, se obtuvieron datos significativos en la subescala falta de realización $(p<0,05)$. En este sentido, la prueba Scheffé que se realizó con posterioridad nos muestra como el profesorado con 29 años o menos, así como quienes tienen edades comprendidas entre los 30 y los 39 , sienten menos realización que los grupos que se encuentran entre los 40 y los 49 años.

Además, se analizó la variable años de experiencia docente, obteniendo en la prueba ANOVA unos resultados diferenciales significativos $(p<0,05)$. Posteriormente, en el análisis de los contrastes realizados observamos como los cuerpos docentes con más experiencia profesional (20 años o más) presentan niveles más bajos de falta de realización que docentes con menos experiencia (10 años o menos). Asimismo, estos grupos educadores con una experiencia más amplia (20 años o más) muestran niveles de burnout menores que el profesorado que tiene entre 11 y 19 años de práctica en la profesión.

Atendiendo al tipo de centro en el que trabajan, las diferencias en el nivel de burnout se interpretan como significativas, únicamente, en la subescala falta de realización $(p<0,05)$. El análisis de los contrastes posteriores, mediante la prueba Scheffé llevada a cabo muestra que el profesorado de los centros públicos tiene un nivel de falta de realización personal más elevado que el que desempeña su labor en los centros privados.

Al realizar el análisis diferencial por nivel de enseñanza se encontraron diferencias significativas $(p<0,05)$ solo entre el profesorado que impartía clases en educación infantil o primaria y el que impartía clases en educación secundaria. Los contrastes posteriores muestran que el profesorado que impartió clases en infantil o primaria presenta un nivel de despersonalización menor que el profesorado que impartió clases en secundaria (ver Tabla 6). 
Tabla 6: Resumen de los descriptivos y ANOVA del burnout en sus diferentes subescalas analizadas

\begin{tabular}{|c|c|c|c|c|c|c|}
\hline \multicolumn{7}{|c|}{ Edad } \\
\hline & & $\mathrm{N}$ & Media & D.T. & $\mathrm{F}$ & Sig. \\
\hline \multirow[t]{4}{*}{ Falta de realización } & 29 años o menos & 230 & 3,67 & 0,466 & 6,738 & 0,024 \\
\hline & 30-39 años & 710 & 3,55 & 0,489 & & \\
\hline & 40-49 años & 660 & 3,40 & 0,576 & & \\
\hline & 50 años o más & 290 & 3,33 & 0,543 & & \\
\hline \multicolumn{7}{|c|}{ Años de experiencia docente } \\
\hline & & $\mathrm{N}$ & Media & D.T. & $\mathrm{F}$ & Sig. \\
\hline \multirow[t]{4}{*}{ Falta de realización } & 29 años o menos & 290 & 3,74 & 0,436 & 4,538 & 0,034 \\
\hline & 30-39 años & 710 & 3,82 & 0,489 & & \\
\hline & 40-49 años & 760 & 3,73 & 0,596 & & \\
\hline & 50 años o más & 130 & 3,71 & 0,523 & & \\
\hline \multirow[t]{4}{*}{ Burnout } & 4 años o menos & 290 & 2,91 & 0,826 & 5,96 & 0,014 \\
\hline & 5-10 años & 710 & 2,90 & 0,763 & & \\
\hline & 11-19 años & 760 & 2,97 & 0,800 & & \\
\hline & 20 años o más & 130 & 2,81 & 0,800 & & \\
\hline \multicolumn{7}{|c|}{ Tipo de centro } \\
\hline & & $\mathrm{N}$ & Media & D.T. & $\mathrm{F}$ & Sig. \\
\hline \multirow[t]{2}{*}{ Falta de realización } & Público & 1540 & 3,89 & 0,441 & 3,74 & 0,019 \\
\hline & Privado & 350 & 3,66 & 0,552 & & \\
\hline \multicolumn{7}{|c|}{ Nivel de enseñanza } \\
\hline & & $\mathrm{N}$ & Media & D.T. & $\mathrm{F}$ & Sig. \\
\hline \multirow[t]{3}{*}{ Despersonalización } & Educación infantil & 270 & 2,03 & 0,312 & 3,935 & 0,001 \\
\hline & Educación primaria & 400 & 2,12 & 0,366 & & \\
\hline & Educación secundaria & 1220 & 2,34 & 0,528 & & \\
\hline
\end{tabular}

Nota: Elaboración propia a partir de Rodríguez y Fernández (2012).

Finalmente, se realizaron análisis diferenciales en función del género, las relaciones personales, el número de hijos e hijas, las áreas o asignaturas impartidas y la situación laboral. Sin embargo, en ninguno de estos casos se encontraron diferencias significativas ni en los niveles del burnout ni en sus tres dimensiones. 
doi: http://dx.doi.org/10.15359/ree.23-2.17

URL: http://www.una.ac.cr/educare

CORREO: educare@una.cr

\section{Conclusiones}

La profesión docente ha adquirido, desde hace años, la caracterización de profesión de riesgo, tanto físico como mental (Moraes, Camargo, Welter y Guisso, 2010; Prieto, 2011; Zamora et al., 2017), como ya la tiene considerada hace décadas la propia OIT. Esta circunstancia se aprecia en las propias valoraciones de los propios grupos de docentes que cumplimentaron el cuestionario en este estudio.

Por todo ello, es necesario adquirir nuevos conocimientos y aptitudes, ya que el paulatino ritmo de cambios de la sociedad y las escuelas actuales hace trascendental una mayor preparación y cualificación (López-Noguero, 2008), así como una evidente labor de prevención en el sector docente basada en a) apoyo emocional, b) apoyo instrumental, c) apoyo informativo, y d) evaluativo.

Abundando sobre los resultados obtenidos destacamos que los valores del Alfa de Cronbach obtenidos en la escala de burnout y en sus tres subescalas se encuentran entre 0,770 y 0,949 , lo que nos indica niveles altos de consistencia. En general, los valores entre 0.7 y 0.8 son 'aceptables', los valores entre 0.8 y 0.9 son 'buenos' y los valores $\geq 0.9$ son 'excelentes' (George y Mallery, 2003). Los resultados encontrados en el análisis de la consistencia interna nos permiten afirmar que la escala de burnout del cuestionario CBP-R es un instrumento fiable y válido para evaluar el burnout en el profesorado.

En la escala de burnout del cuestionario CBP-R se encontró un nivel medio de burnout (88,3\% de docentes se ubicó dentro del rango esperado). También se encontró un nivel medio de burnout en cada una de las subescalas que la componen: agotamiento emocional (73\% de docentes se ubicó dentro del rango esperado), despersonalización (83\% de docentes se ubicó dentro del rango esperado) y falta de realización (86,2\% de docentes se ubicó dentro del rango esperado).

Las mayores frecuencias obtenidas en la escala de burnout y en sus subescalas se ubicaron en los rangos esperados (nivel medio de burnout); se obtuvieron frecuencias de puntajes mayores al esperado (nivel alto) en la escala de burnout y en dos de sus subescalas (agotamiento emocional y despersonalización); solo se obtuvieron frecuencias ubicadas en el rango menor al esperado (nivel bajo) en la subescala falta de realización.

En el estudio se encontraron diferencias significativas en el nivel de burnout de docentes o en alguna de sus dimensiones en función de la edad, los años de experiencia docente, el tipo de centro y el nivel de enseñanza.

En cuanto a la variable 'edad', se encontraron diferencias significativas en la subescala falta de realización. En esta subescala, los grupos docentes de menor edad mostraron mayores niveles de falta de realización que los de mayor edad. 
Con respecto a la variable 'años de experiencia docente', se obtuvieron diferencias significativas en la subescala falta de realización y en la escala de burnout. En la subescala falta de realización, el profesorado con mayor antigüedad presentó menores niveles de burnout que el de menor antigüedad. En este sentido, se aprecia cómo los síntomas del burnout relacionados con la falta de realización se presentan de manera más aguda entre docentes más jóvenes, por lo que podríamos inferir que la experiencia en el ámbito educativo puede suponer un "cortafuegos" ante este tipo de problemática, ya que le podría permitir tomar más distancia con las dificultades, adaptarse a situaciones cambiantes $y$, por tanto, manejar mejor las situaciones de estrés.

En referencia a la variable 'tipo de centro', se encontraron diferencias significativas en la subescala falta de realización. En esta subescala, los cuerpos docentes de los centros públicos mostraron un nivel mayor de falta de realización personal que los de los centros privados. Así, los datos parecen indicar que docentes que desarrollan su labor en centros públicos sufren con más agudeza una falta de realización personal que quienes trabajan en centros privados. Esto puede ser debido a diversas circunstancias como la presión exigida por los objetivos establecidos desde la dirección provincial, la alta conflictividad del alumnado en algunos de estos centros, la falta de recursos, la masificación de las aulas o las características de los entornos multiculturales.

En cuanto a la variable 'nivel de enseñanza', se obtuvieron diferencias significativas en la subescala despersonalización. En esta subescala, docentes que impartieron clases en infantil o primaria presentaron un nivel de despersonalización menor que quienes impartieron clases en secundaria. De acuerdo con esto, podemos deducir que la edad estudiantil influye en la aparición de esta problemática asociada a la despersonalización de docentes, ya que el estudiantado adolescente puede llegar a presentar unas características conductuales que resultan más difíciles de gestionar desde una perspectiva socioeducativa.

En definitiva, podemos concluir señalando que la información obtenida en esta investigación, y su posterior análisis e interpretación contribuyen al desarrollo del conocimiento científico sobre el síndrome de burnout. Deigual forma, es necesario que las futuras investigaciones ahonden en los diversos aspectos que influyen en la manifestación del burnout en docentes. Tal y como señalan Rodríguez y Fernández (2012, p. 274), es apropiado "profundizar en el modelo ... explicativo del síndrome que interrelaciona las tres dimensiones del burnout entre sí y en relación con dimensiones o factores externos al mismo ... o factores inherentes a la persona".

\section{Referencias}

Bilge, F. (2006). Examining the burnout of academics in relation to job satisfaction and other factors. Social Behavior and Personality, 34(9), 1151-1160. doi: https://doi.org/10.2224/ sbp.2006.34.9.1151 
doi: http://dx.doi.org/10.15359/ree.23-2.17

URL: http://www.una.ac.cr/educare

CORREO: educare@una.cr

Botella, L. L., Longás, J. y Gómez, A. M. (2007). La construcción social del burnout en la profesión docente. Aloma, 21, 271-296. Recuperado de https://www.raco.cat/index.php/Aloma/ article/view/89978/115137

Boyd, B. J. y Schneider N. (2014). Perceptions of the work environment and burnout in Canadian child care providers. Journal of Research in Childhood Education, Association for Childhood Education International, 11(2), 171-180. doi: https://doi.org/10.1080/02568549709594704

Cárdenas, M., Méndez, L. M. y González, M. T. (2014). Desempeño, estrés, burnout y variables personales de los docentes universitarios. Educere, 18(60), 289-302. Recuperado de http:// www.redalyc.org/articulo.oa?id=35631743009

Extremera, N., Fernández-Berrocal, P. y Durán, A. (2003). Inteligencia emocional y burnout en profesores. Encuentros en Psicología Social, 1, 260-265. Recuperado de http://emotional. intelligence.uma.es/documentos/PDF5burnout en profesores.pdf

Fidalgo, M. (2005). NTP705:Síndrome de estarquemado por el trabajo o "burnout" (II): Consecuencias, evaluación y prevención. Madrid:Ministerio de Trabajo y Asuntos Sociales e Instituto Nacional de Seguridad e Higiene en el Trabajo. Recuperado de http://www.insht.es/InshtWeb/ Contenidos/Documentacion/FichasTecnicas/NTP/Ficheros/701a750/ntp 705.pdf

Freudenberger, H. J. (1974). Staff burn-out. The Journal of Social Issues, 30(1), 159-165. doi: https:// doi.org/10.1111/j.1540-4560.1974.tb00706.x

Fuertes, C., Aranda, G. y Arroyo, M. P. (2016). Comunicación y mindfulness como prevención del burnout. Anales del Sistema Sanitario de Navarra, 39(2), 331-333. Recuperado de http:// scielo.isciii.es/pdf/asisna/v39n2/20 cartas5.pdf

George, D. y Mallery, P. (2003). SPSS for windows step by step: A simple guide and reference. Fourth edition (11.0 update). Boston: Allyn \& Bacon. Recuperado de https://wps.ablongman.com/ wps/media/objects/385/394732/george4answers.pdf

Gil, P. R. (2001). El síndrome de quemarse por el trabajo (síndrome de burnout): Aproximaciones teóricas para su explicación y recomendaciones para la intervención. Revista PsicologiaCientífica.com, 3(5), 1-5. Recuperado de https://www.researchgate.net/ profile/Pedro Gil-Monte/publication/242114408 El sindrome de quemarse por el trabajo sindrome de burnout aproximaciones teoricas para su explicacion $y$ recomendaciones para la intervencion/links/0deec534528db5d80e000000.pdf

Gil, P. R. (2003). El síndrome de quemarse por el trabajo (síndrome de Burnout) en profesionales de enfermería. Revista Eletrônica InterAção Psy, 1(1), 19-33.

Góngora, F., Pérez, M. L. y Torres, M. R. (2016). Síndrome de burnout y enfermería: Diagnóstico y prevención. En F. R. Jiménez (Dir.) y F. J. López (Ed.), Gestión pública de la salud (pp. 121126). Madrid: Asociación Cultural y Científica Iberoamericana. 
Revista Electrónica Educare (Educare Electronic Journal) EISSN: 1409-4258 Vol. 23(2) MAYO-AGOSTO, 2019:1-20

doi: http://dx.doi.org/10.15359/ree.23-2.17

URL: http://www.una.ac.cr/educare

CORREO: educare@una.cr

Gutiérrez, M., Atienza, M. y García-Moya, R. (2015). Técnicas de apoyo psicológico y social en situaciones de crisis. Madrid: Paraninfo.

Instituto de Estadística y Cartografía de Andalucía. (2016). Sistema de información multiterritorial de Andalucía (SIMA). Sevilla: Autor. Recuperado de http://www.juntadeandalucia.es/ institutodeestadisticaycartografia/iea/consultasActividad.jsp?CodOper $=104 \&$ sub $=38120$

Kroupis, I., Koustessis, T., Kouli, O., Tzetzis, G., Derri, V. y Mavrommatis, G. (2017). Job satisfaction and burnout among Greek P.E. teachers. A comparison of educational sectors, level and gender. Cultura, ciencia y deporte. Revista de ciencias de la actividad física y del deporte de la Universidad Católica de San Antonio, 12(34), 5-14. doi: https://doi.org/10.12800/ccd. v12i34.827

Langher, V., Caputo, A y Ricci, M. E. (2017). The potential role of perceived support for reduction of special education teachers' burnout. International Journal of Educational Psychology, 6(2), 120-147. doi: https://doi.org/10.17583/ijep.2017.2126

Ledesma, C. A. (2015). Síndrome de burnout en el personal asistencial de la Clínica del Hospital Hermilio Valdizán (Tesis doctoral). Universidad Ricardo Palma, Perú. Recuperado de http:// cybertesis.urp.edu.pe/bitstream/urp/580/1/ledesma ca.pdf

López-Noguero, F. (2008). Hacia un espacio europeo de aprendizaje para toda la vida. Evolución y desarrollo de la educación permanente en la Unión Europea. Pedagogía social. Revista Interuniversitaria, 15, 123-135. doi: https://doi.org/10.7179/PSRI 2008.15.10

López-Noguero, F. (2015). Los nuevos retos de la investigación en educación para la salud. En J. A. Morón. (Eds), Investigar e intervenir en educación para la salud (pp. 31-44). Madrid: Narcea.

Mameli, C y Molinari, L. (2017). Teaching interactive practices and burnout. A study on Italian teachers. European journal of psychology of education, 32(2), 219-234. Doi: https://doi. org/10.1007/s10212-016-0291-z

Manassero, M. A., Vázquez, Á., Ferrer, V. A., Fornés, J. y Fernández, M. (2003). Estrés y burnout en la enseñanza. Palma de Mallorca: Edicions UIB.

Moraes, R., Camargo, J., Welter, M. M. y Guisso. L. (2010). Saúde docente, condições e carga de trabalho. Revista Electrónica de Investigación y Docencia. 4, 147-160. Recuperado de http:// www.ujaen.es/revista/reid/revista/n4/REID4art8.pdf

Moreno, B., Garrosa, E.y González, J. L. (2000).La evaluación del estrés y el burnout del profesorado: El CBP-R. Revista de Psicología del Trabajo y de las Organizaciones, 16(2), 151-171. 
doi: http://dx.doi.org/10.15359/ree.23-2.17

URL: http://www.una.ac.cr/educare

CORREO: educare@una.cr

Pena, M. y Extremera, N. (2012). Inteligencia emocional percibida en el profesorado de primaria y su relación con los niveles de burnout e ilusión por el trabajo. Revista de Educación, 359, 604-627. Recuperado de https://www.researchgate.net/profile/Natalio Extremera/ publication/312463196 Inteligencia emocional percibida en profesorado de primaria y su relacion con los niveles de burnout e ilusion por el trabajo engagement/links/5a7820a00f7e9b41dbd26907/Inteligencia-emocional-percibida-enprofesorado-de-primaria-y-su-relacion-con-los-niveles-de-burnout-e-ilusion-por-eltrabajo-engagement.pdf

Pines A. y Aronson, E. (1998). Career burnout: Causes and cures. New York, NY: Free Press.

Prieto, M. (2011). Estrés y ansiedad en profesores. Crítica, 61(974), 42-45. Recuperado de http://www.revista-critica.com/administrator/components/com avzrevistas/pdfs/ e80b23dbdecd08c1871d57a0507c0554-974-Emociones-que-nos-rompen-ansiedad-ydepresi--n---jul.ago.2011.pdf

Rodríguez, J. M. y Fernández, M. J. (2012). El síndrome de burnout en el profesorado de secundaria y su relación con variables personales y profesionales. Revista Española de Pedagogía, 70(252), 259-277. Recuperado de https://revistadepedagogia.org/wp-content/ uploads/2012/12/252-07.pdf

Rodríguez, J. M y Fernández, M. J. (2017). The effect of interpersonal relationships on burnout syndrome in Secondary Education teachers. Psicothema, 29(3), 370-377.

Shami, R., Tare, M y Taran, H. (2017). Identifying the relationship among teacher's mental health and emotional intelligence and their burnout. Independent Journal of Management \& Production, 8(1), 124-143. doi: https://doi.org/10.14807/ijmp.v8i1.513

Sierra, R. (1987). Técnicas de investigación social. Madrid: Paraninfo.

Solera, E., Gutiérrez, S. y Palacios-Ceña, D. (2017). Evaluación psicológica del síndrome de burnout en profesores de educación primaria en la comunidad de Madrid: comparación entre centros públicos y concertados. Universitas Psychologica, 16(3). 1-9. doi: ttps://doi. org/10.11144/Javeriana.upsy16-3.epsb

Zamora-Díaz, W.-J., Cobos-Sanchiz, D. y López-Noguero, F. (2017). Condiciones sociales y salud laboral del profesorado nicaragüense de secundaria. Revista de Pedagogía, 38(103), 192208. Recuperado de http://www.redalyc.org/pdf/659/65954978010.pdf

Zamora-Díaz, W. J., López-Noguero, F. y Cobos-Sanchiz, D. (2016). Realidades del empleo docente en Nicaragua. Revista Electrónica de Investigación Educativa, 18(2), 191-205. Recuperado de https://redie.uabc.mx/redie/article/view/1127/1456 